J Neurooncol. 2011 July ; 103(3): 791-796. doi:10.1007/s11060-010-0453-9.

\title{
Progressive multifocal leukoencephalopathy in a patient with glioblastoma
}

\author{
Jing Wu, \\ Department of Neuro-Oncology, The University of Texas MD Anderson Cancer Center, Houston, \\ TX 77030, USA \\ Lauren A. Langford, \\ Department of Pathology, The University of Texas MD Anderson Cancer Center, Houston, TX \\ 77030, USA \\ Dawid Schellingerhout, \\ Department of Radiology, The University of Texas MD Anderson Cancer Center, Houston, TX \\ 77030, USA \\ Nandita Guha-Thakurta, \\ Department of Radiology, The University of Texas MD Anderson Cancer Center, Houston, TX \\ 77030, USA

\section{Sudhakar Tummala,} \\ Department of Neuro-Oncology, The University of Texas MD Anderson Cancer Center, Houston, \\ TX 77030, USA
}

Jeffrey S. Weinberg, and

Department of Neurosurgery, The University of Texas MD Anderson Cancer Center, Houston, TX 77030, USA

\section{Vinay K. Puduvalli}

Department of Neuro-Oncology, The University of Texas MD Anderson Cancer Center, Houston, TX 77030, USA

\section{Abstract}

Malignant gliomas are aggressive malignancies which inevitably recur despite multimodality treatment. In a subset of patients who are longer term survivors of this disease, progressive radiologic worsening can also occur from late effects of radiation rather than recurrent tumor, a differential diagnosis that is commonly considered in this setting. However, other causes for radiologic progression are not as well recognized and could potentially confound management leading to incorrect treatment decisions. Progressive multifocal leukoencephalopathy (PML) is a rare infectious demyelinating disease of the central nervous system seen primarily in immunocompromised patients, the early diagnosis and treatment of which remains a challenge. Here, we report a case of a long term survivor with glioblastoma whose diagnostic and therapeutic 
management was confounded by the development of PML. We review the radiological features and clinical course of this patient to highlight the dramatic neurological course in the setting of a highly malignant tumor, and emphasize the unusual changes in diffusion weighted images, and the need for clinical suspicion for early diagnosis of PML.

\section{Keywords}

Progressive multifocal; leukoencephalopathy; Glioblastoma; Immunosuppression; Differential diagnosis

\section{Introduction}

Recent advances in treatment options for patients with glioblastoma have provided definite, albeit modest improvement in progression-free and overall survival. After upfront therapy with chemo-radiation, surveillance for disease activity is done by periodic clinical and radiological evaluation; tumor progression is most commonly detected as new or progressive contrast-enhancement on MRI scans. In long term survivors of this disease, the appearance of contrast enhancing foci may also be the result of disruption of the blood-brain barrier and injury to normal brain tissue due to late effects of radiation therapy, which is a well recognized and commonly considered differential diagnosis in this setting. However, the distinction between tumor and treatment effects is a diagnostic challenge which can delay treatment decisions. Contrast enhancing lesions can also occasionally develop from other processes which may be mistaken for tumor progression or treatment effect and which can confound diagnosis and clinical management. Because such processes are rare, they are often not considered in the differential diagnosis of new radiologic changes in patients with malignant brain tumors resulting in delays in diagnosis and management of the same.

Progressive multifocal leukoencephalopathy (PML) is an infectious disease of central nervous system (CNS) caused by the JC virus which results in patchy demyelination and associated progressive neurological symptoms. This entity was first described by Astrom et al. in 1958 based on the histological features of white matter lesions from patients with lymphoma [1]. The detection of polyoma virus particles in the nuclei of oligodendrocytes in 1965 and the isolation of this virus from a patient with PML in 1971 established the infectious nature of this entity [2].

Asymptomatic JC virus infection occurs in about $80-90 \%$ of healthy adults who are seropositive for the virus [3]. Although the exact mode of transmission of JC virus is uncertain, it is thought to spread via an oro-fecal route [4]. After the initial infection of the tonsillar tissue and subsequent systemic spread [5], the virus can remain quiescent in the epithelial cell of the kidneys and lymphoid tissue for the life of the individual [6]. The virus can be reactivated in the setting of immunodeficiency, at which time it targets oligodendrocytes and astrocytes to cause cytolytic destruction of the myelin, resulting in fatal disease. PML has been reported in the setting of immunosuppression as a result of human immunodeficiency virus (HIV) infection [7], organ transplantation [8] or inherited immunodeficiencies [9]. It has also been reported in patients with multiple sclerosis and Crohn's disease receiving natalizumab treatment. There have been PML cases were reported in patients with 
hematological malignancy, especially in chronic lymphocytic leukemia and B cell lymphoma after receiving Rituximab and cyclophosphamide, doxorubicin, vincristine and prednisone (R-CHOP) [10] or bone marrow transplant [11]. However, to our knowledge, there has been no PML case reported in patients with malignant gliomas.

\section{Case history}

A 58 year-old otherwise healthy gentleman presented with impaired speech and word finding difficulty and was found to have a left frontal enhancing mass by brain MRI. The lesion was gross totally resected and confirmed to be a glioblastoma by pathological exam. He underwent radiation therapy to the left frontal tumor region with a $2 \mathrm{~cm}$ margin to a total dose of 60 Gray in 30 fractions along with concurrent temozolomide (TMZ) at $75 \mathrm{mg} / \mathrm{m}^{2} /$ day. Adjuvant chemotherapy was initiated with dose dense TMZ $\left(100 \mathrm{mg} / \mathrm{m}^{2}\right.$ on days $1-7$ and $15-21)$ and isotretinoin $\left(100 \mathrm{mg} / \mathrm{m}^{2}\right.$ on days $1-21$ days of a 28 day cycle). After an early complication of colon perforation likely related to steroid therapy and isotretinoin, which required an emergent colostomy, treatment was well tolerated and the patient remained stable clinically and radiologically for approximately 2 years. At this time, a routine brain MRI revealed a new enhancing lesion near the left frontal surgical cavity which was resected and confirmed to be recurrent glioblastoma. After recovery from the surgery, he was enrolled in a randomized clinical trial of lomustine plus either cediranib or placebo. Prior to initiation of therapy, the patient reported generalized weakness and fatigue; more intriguingly, he complained of isolated left-sided lower extremity weakness, which was ipsilateral to the tumor. Electrophysiological studies showed reduced activation patterns on the EMG that suggested central process with no evidence for peripheral nerve injury. A spine MRI scan was unremarkable; however, a brain MRI showed minimal T2 fluidattenuated inversion recovery (FLAIR) hyperintensity along the medial aspect of the right pre-central gyrus involving subcortical white matter without mass effect and sparing the gray matter (Fig. 1A, upper panel i, ii); mild diffusion restriction on diffusion weighted images (DWI) was also seen (Fig. 1A, lower panel) not associated with contrast-enhancement (Fig. $1 \mathrm{~A}$, right panel).

Due to the subtle nature of the MRI changes and the presence of histologically proven recurrent tumor, chemotherapy was initiated; however, the patient developed severe myelosuppression after the first cycle of treatment requiring discontinuation of treatment. After partial recovery of blood counts, a decision was made to initiate treatment with irinotecan and bevacizumab. However, the patient's left lower extremity weakness progressed and a repeat MRI scan showed that while the tumor region in the left frontal region had stable enhancement with corresponding FLAIR hyperintensity, the lesion in the right frontoparietal subcortical region increased in size (Fig. 1A, upper panel, iii, iv) and while remaining non-enhancing post-contrast, developed more prominent restricted diffusion on DWI sequences (Fig. 1A, lower panel, iii, iv). Over the next 8 weeks, the left-sided weakness gradually progressed despite a course of steroid therapy to the point that the patient was unable to ambulate even with assistance. MRI studies revealed that the lesion increased in size with dramatically increased diffusion restriction in DWI sequences (Fig. 1B). At this point, the possibility of progressive treatment effect modified by antiangiogenic therapy was initially considered; however, given that the lesion was at the periphery of the 
radiation field and had characteristic radiological features, the alternative diagnosis of PML was also considered. EEG showed rare epileptiform discharges arising from right central area with no clinical seizures. Expected focal dysfunction was noted in the left frontal region (Fig. 1C). The patient was offered the option of a surgical biopsy of the right frontal lesion for definitive tissue diagnosis. A lumbar puncture was also considered for CSF analysis for evidence of JC virus infection. However, these procedures were deferred because the patient worsened clinically. Shortly before the scheduled brain biopsy, because the patient had rapid clinical deterioration with progressive encephalopathy, the family and the medical team reached a consensus decision to forego the biopsy and transition to supportive care and home hospice.

After the patient died, the family consented to an autopsy limited to the brain. Gross examination showed the old surgical cavity in the left frontal region. On cut section, the white matter in both hemispheres, as well as in the brain stem and cerebellum, contained a myriad of small, punctate lesions. In areas with large numbers of lesions (Fig. 2A), the lesions coalesced to form an area of softening suggestive of necrosis. The extent of softening was particularly prominent in the right centrum semiovale and corpus callosum. Microscopic examination of the hematoxylin and eosin stained sections showed the lesions to be rarefied neuropil filled with macrophages and with loss of the normal myelin tapestry. Large, hyperchromatic cells with "glassy" nuclei were scattered at the edges of these demyelinated lesions. (Fig. 2B) Areas of demyelination were observed throughout the white matter of the brain. (Fig. 2C) The nuclei of the large cells, interpreted to be oligodendrocytes, were filled with viral particles that were identified with immunohistochemistry to be JC virus. (Fig. 2D).

\section{Discussion}

PML is a rare demyelinating disease in CNS which is characterized by multifocal lesions restricted to the white matter with a rapidly progressive clinical course uniformly resulting in a fatal outcome. Seropositivity of JC virus is common in the majority of the population but clinically evident disease is seldom seen in immunocompetent patients. However, in the setting of immunodeficiency, the virus can be reactivated and can replicate in the oligodendroglial cells causing clinical PML. In a study of 61 patients with PML conducted at the Massachusetts General Hospital, 48 had acquired immunodeficiency syndrome (AIDS), 8 had hematological cancer, 3 had bone marrow transplant and 1 had dermatomyositis [6]. In HIV infected patients, about 5-8\% developed PML due to immunosuppression and the risk of development of the disease increases with increased viral load [12]. In the non-AIDS population, PML often develops as result of immunosuppression due to chemotherapy or immunosuppressants.

PML is considered in the immunosuppressed patient due to the concomitant development of the neurological symptoms and neuroimaging findings which are characteristic but not confirmatory of this condition. A definitive diagnosis is established by brain biopsy to demonstrate the presence of JCV in the brain tissue. Alternatively, demonstration of JCV DNA in the cerebrospinal fluid by polymerase chain reaction (PCR) is also confirmatory in 
the appropriate clinical setting with a sensitivity and specificity of 74 and $96 \%$ respectively [13].

In our patient, consideration of PML was confounded by several factors. The history of prior chemo-radiation therapy and the unifocal nature of the new radiological changes in MRI findings initially made the possibility of delayed effects of radiation therapy a more likely etiology for the new lesion; however, a review of the radiation plan showed that the lesion had occurred in the periphery of the treatment field making this less likely. An invasive focus of recurrent disease due to subependymal spread to the left hemisphere was also considered. However, the development of rapidly progressive neurological deficit and of particular significance, the presence of imaging changes sharply confined to the white matter resulted in consideration of PML. Although the MRI study prior to the initial presentation of the left leg weakness showed only trace linear T2/FLAIR hyperintensity in the right pre-central gyrus, the lesion progressed with involvement of the subcortical U-fibers without mass effect or contrast-enhancement. Of particular note, the DWI sequence showed restricted diffusion not corresponding to a specific vascular territory making an ischemic event unlikely. The lesion had also been present prior to bevacizumab therapy and progressed after its discontinuation making it unlikely to be treatment related and suggesting an alternative etiology.

Restricted diffusion is characteristically seen due to intracellular edema resulting from acute cell injury such as in an ischemic infarct; however, this can also be seen in processes such as PML [14]. In this patient, the brain MRIs evolved over time to show radiological features more characteristics of the PML; such findings were reported in a previous study [15] in which brain MRIs in patients with PML showed T2/FLAIR hyperintensity, with decreased T1 signal intensity, and restricted diffusion on DWI without contrast-enhancement or mass effect.

Lymphopenia is commonly seen in patients receiving chemotherapy and has been associated with opportunistic infections. CD4+ and CD8+ T lymphopenia and B lymphocyte depletion are reported as risk factors in developing PML [16]. Lymphopenia was noted in our patient when the first imaging abnormality with an absolute lymphocyte count (ALC) of 240 although lymphocyte sub-typing was not available. When the clinical symptoms worsened, the ALC further decreased to 80. The combination of lymphopenia with ongoing and necessary steroid and chemotherapy in our patient likely contributed to the immunosuppressed state that facilitated development of PML.

Features that were atypical for PML in this case included the unifocal development of the lesion and the relatively slow progression of disease ( $>6$ months from initial symptoms) when compared with the rapid progression of typical PML. While it is tempting to speculate that the chemotherapy and antiangiogenic therapy given during the initial period of the course of PML may have decelerated its progression, the relationship of the disease to ongoing therapy remains unknown.

Management of PML remains uncertain and no definitive treatments have emerged; hence, it remains an inexorably progressive disease for which there is no cure. In patients with AIDS 
related PML, HAART has been relatively effective in reducing the incidence of the condition [17]. Cytarabine has been tested in both AIDS associated and non-AIDS associated PML. However, it was not found to improve the prognosis in AIDS associated PML in a randomized controlled trial involving 57 patients [18]. In non-AIDS related PML patients, several studies have demonstrated improvement with cytarabine [19]. In a retrospective study, $37 \%$ of patients with non-AIDS related PML achieved disease stabilization with cytarabine treatment. However, nearly all patients did not recover from the neurological deficit and most died with a year after onset of the disease. In our patient, there was no opportunity to initiate this treatment in the setting of myelosuppression and rapid progression of disease.

In summary, we present a rare case of PML in patient with glioblastoma. While the incidental detection of glioma in patients who died of PML has been reported, to our knowledge, this is the first case of a patient with glioblastoma under active therapy who developed PML. The occurrence of diffusion restriction in MRI scans in a predominantly subcortical white matter based lesion, distinct from the appearance of the tumor, is a sign that should raise suspicion for the possibility of PML in the setting of immunosuppression even if the lesion is unifocal. In such cases, diagnostic tests including CSF studies and if necessary, surgical biopsy must be undertaken. Additional studies are needed to verify the specificity of the radiological findings reported in this case including diffusion restriction in identifying PML non-invasively and early in its course; the development of effective treatment for this condition remains a more formidable challenge.

\section{References}

1. Astrom KE, Mancall EL, Richardson EP Jr. Progressive multifocal leukoencephalopathy; a hitherto unrecognized complication of chronic lymphatic leukaemia and Hodgkin's disease. Brain. 1958; 81:93-111. [PubMed: 13523006]

2. Padgett BL, Walker DL, ZuRhein GM, Eckroade RJ, Dessel BH. Cultivation of papova-like virus from human brain with progressive multifocal leucoencephalopathy. Lancet. 1971; 1:1257-1260. [PubMed: 4104715]

3. Weber T, Trebst C, Frye S, Cinque P, Vago L, Sindic CJ, Schulz-Schaeffer WJ, Kretzschmar HA, Enzensberger W, Hunsmann G, Luke W. Analysis of the systemic and intrathecal humoral immune response in progressive multifocal leukoencephalopathy. J Infect Dis. 1997; 176:250-254. [PubMed: 9207375]

4. Maginnis MS, Atwood WJ. JC virus: an oncogenic virus in animals and humans? Semin Cancer Biol. 2009; 19:261-269. [PubMed: 19505654]

5. Kato A, Kitamura T, Takasaka T, Tominaga T, Ishikawa A, Zheng HY, Yogo Y. Detection of the archetypal regulatory region of JC virus from the tonsil tissue of patients with tonsillitis and tonsilar hypertrophy. J Neurovirol. 2004; 10:244-249. [PubMed: 15371154]

6. Koralnik IJ, Schellingerhout D, Frosch MP. Case records of the Massachusetts General Hospital. Weekly clinicopathological exercises. Case 14-2004. A 66 year-old man with progressive neurologic deficits. N Engl J Med. 2004; 350:1882-1893. [PubMed: 15115835]

7. Morriss MC, Rutstein RM, Rudy B, Desrochers C, Hunter JV, Zimmerman RA. Progressive multifocal leukoencephalopathy in an HIV-infected child. Neuroradiology. 1997; 39:142-144. [PubMed: 9045978]

8. Shitrit D, Lev N, Bar-Gil-Shitrit A, Kramer MR. Progressive multifocal leukoencephalopathy in transplant recipients. Transpl Int. 2005; 17:658-665. [PubMed: 15616809]

9. Hecht JH, Glenn OA, Wara DW, Wu YW. JC virus granule cell neuronopathy in a child with CD40 ligand deficiency. Pediatr Neurol. 2007; 36:186-189. [PubMed: 17352955] 
10. Yokoyama H, Watanabe T, Maruyama D, Kim SW, Kobayashi Y, Tobinai K. Progressive multifocal leukoencephalopathy in a patient with B-cell lymphoma during rituximab-containing chemotherapy: case report and review of the literature. Int J Hematol. 2008; 88:443-447. [PubMed: 18855101]

11. Chowdhary S, Chamberlain M. A progressive neurologic disorder with multiple CNS lesions: a neuroimaging clinicopathologic correlation. Progressive multifocal leukoencephalopathy (PML). J Neuroimaging. 2008; 18:340-344. [PubMed: 18819185]

12. Berger JR, Concha M. Progressive multifocal leukoencephalopathy: the evolution of a disease once considered rare. J Neurovirol. 1995; 1:5-18. [PubMed: 9222338]

13. Fong IW, Britton CB, Luinstra KE, Toma E, Mahony JB. Diagnostic value of detecting JC virus DNA in cerebrospinal fluid of patients with progressive multifocal leukoencephalopathy. $\mathrm{J}$ Clin Microbiol. 1995; 33:484-486. [PubMed: 7714213]

14. Major EO, Amemiya K, Tornatore CS, Houff SA, Berger JR. Pathogenesis and molecular biology of progressive multifocal leukoencephalopathy, the JC virus-induced demyelinating disease of the human brain. Clin Microbiol Rev. 1992; 5:49-73. [PubMed: 1310438]

15. Post MJ, Yiannoutsos C, Simpson D, Booss J, Clifford DB, Cohen B, McArthur JC, Hall CD. Progressive multifocal leukoencephalopathy in AIDS: are there any MR findings useful to patient management and predictive of patient survival? AIDS clinical trials group, 243 team. AJNR Am J Neuroradiol. 1999; 20:1896-1906. [PubMed: 10588116]

16. Carson KR, Focosi D, Major EO, Petrini M, Richey EA, West DP, Bennett CL. Monoclonal antibody-associated progressive multifocal leucoencephalopathy in patients treated with rituximab, natalizumab, and efalizumab: a review from the research on adverse drug events and reports (RADAR) project. Lancet Oncol. 2009; 10:816-824. [PubMed: 19647202]

17. Dworkin MS. A review of progressive multifocal leukoencephalopathy in persons with and without AIDS. Curr Clin Top Infect Dis. 2002; 22:181-195. [PubMed: 12520654]

18. Hall CD, Dafni U, Simpson D, Clifford D, Wetherill PE, Cohen B, McArthur J, Hollander H, Yainnoutsos C, Major E, Millar L, Timpone J. Failure of cytarabine in progressive multifocal leukoencephalopathy associated with human immunodeficiency virus infection. AIDS clinical trials group 243 team. N Engl J Med. 1998; 338:1345-1351. [PubMed: 9571254]

19. Bauer WR, Turel AP Jr, Johnson KP. Progressive multi-focal leukoencephalopathy and cytarabine. Remission with treatment. JAMA. 1973; 226:174-176. [PubMed: 4740910] 
A
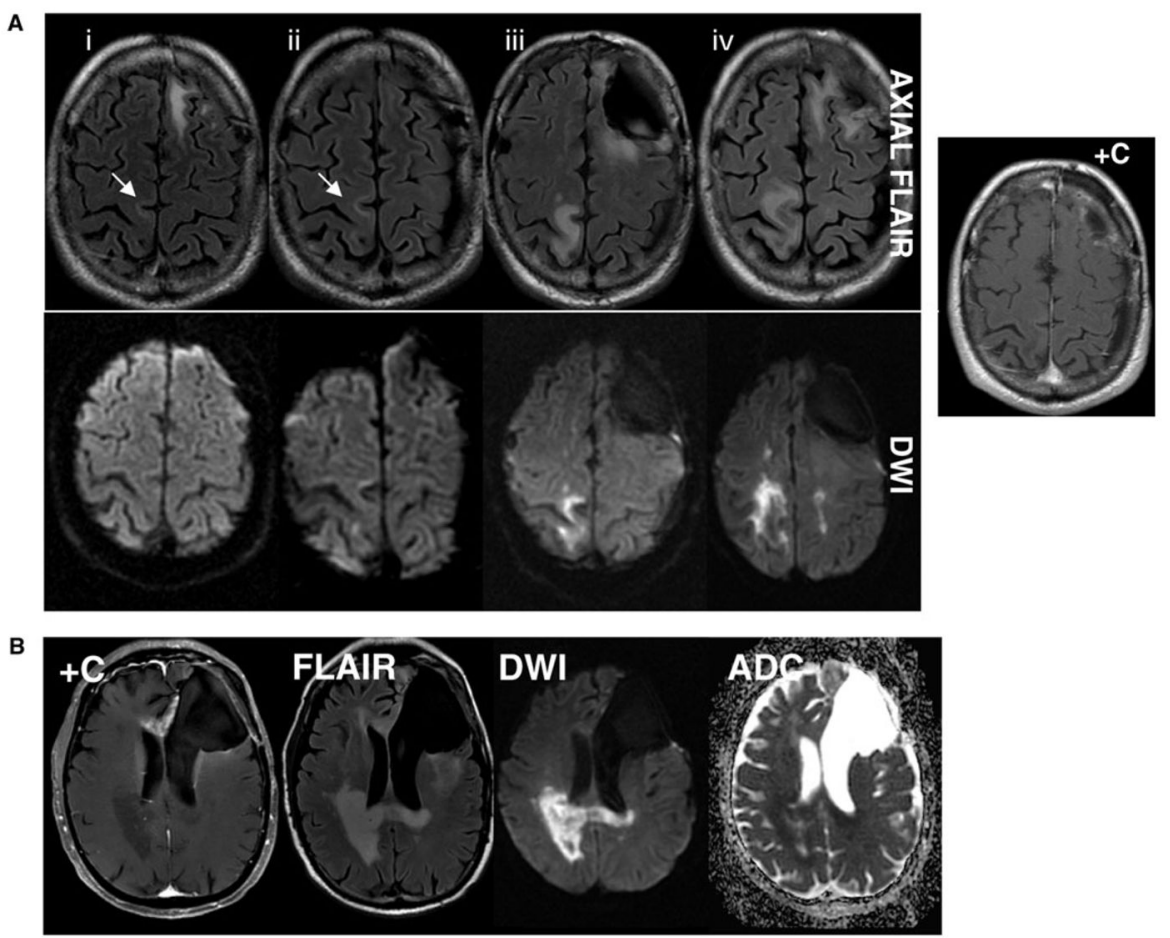

C

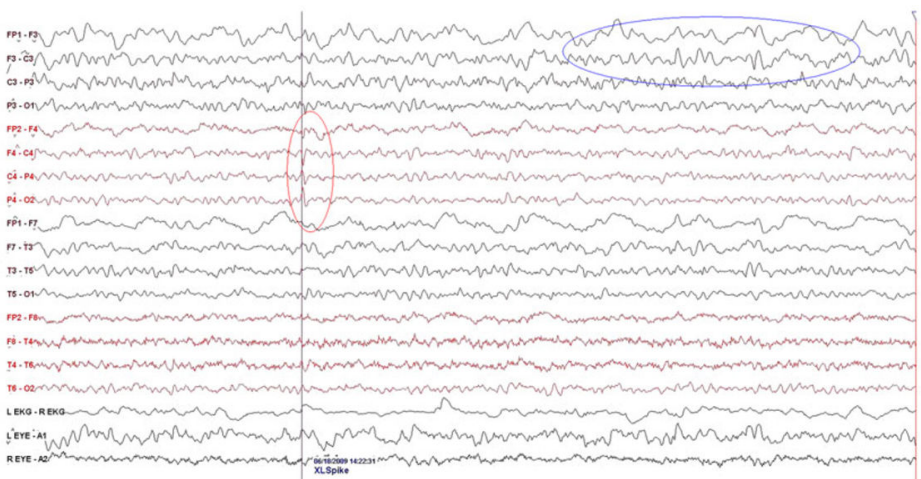

Fig. 1.

A Axial MRI images demonstrate increased signal on the diffusion image (lower panel) corresponding to the white matter FLAIR hyperintensity in the right paracentral lobule (upper panel) without corresponding contrast-enhancement (right panel) over 7 months of the time, which suggests the progression of the lesion. The subtle subcortical FLAIR hyperintensity in the initial MRI scans is indicated (arrows). B Follow-up MR imaging demonstrates the original tumor location in the anterior corpus callosum and adjacent left anteromedial frontal lobe as an enhancing region with FLAIR hyperintensity but without diffusion restriction; in contrast, the right parieto-occipital lesion and adjacent splenium of the corpus callosum shows hyperintense signals in the DWI sequence with decreased signals on ADC map, corresponding to FLAIR hyperintensity and without contrast-enhancement involving the posterior periventricular white matter and the splenium of the corpus callosum. C EEG obtained after patient became intermittently encephalopathic; focal slowing is seen 
in the left frontal site of known tumor (upper oval) and rare epileptiform discharges are evident in the right central region corresponding to the parasagital lesion (lower oval) 

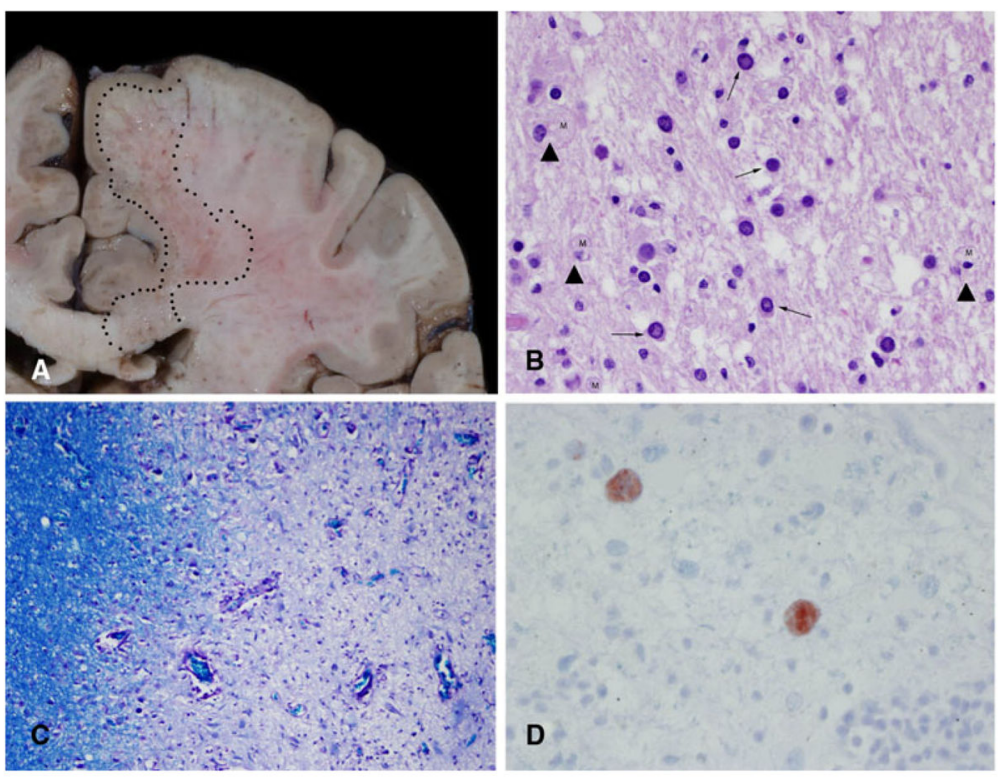

Fig. 2.

Postmortem examination areas of softening and necrosis in the white matter. Large areas of softening in the frontal lobe and corpus callosum are delineated by the dotted areas (A). Hematoxylin and eosin stained sections showed demyelinated regions containing macrophages (arrowhead) and large, atypical virus-laden cells (arrows). $\times 200$ (B). Special stains for myelin (LFB-PAS) show the discrete nature of the lesions. The left side of the image shows intact myelin. The right side of the image is much less dense with loss of most of the myelin. $\times 100(\mathbf{C})$.

Immunohistochemistry preparations for JC virus label the cell nuclei filled with viral particles. $\times 400(\mathbf{D})$ 